\title{
COLANGIOPANCREATOGRAFIA ENDOSCÓPICA RETRÓGRADA TRANSGÁSTRICA LAPAROSCÓPICA APÓS CIRURGIA BARIÁTRICA
}

\author{
LAPAROSCOPIC TRANSGASTRIC ENDOSCOPIC RETROGRADE \\ CHOLANGIOPANCREATOGRAPHY AFTER BARIATRIC SURGERY
}

\author{
Alcides José Branco Filho, TCBC-PR ${ }^{1}$; Rafael William Noda ${ }^{2}$; William Kondo, ACBC-PR ${ }^{1}$; \\ Marco Aurélio de George'; Marlon Rangel, TCBC-PR'
}

\section{INTRODUÇÃO}

O número de pacientes submetidos à cirurgia bariátrica aumentou dramaticamente nos últimos anos, uma vez que a maioria das opções de tratamento conservador para a obesidade mórbida (dieta, exercício físico, alterações comportamentais e medicações) não é capaz de manter uma perda de peso efetiva a longo prazo $^{1}$.

Atualmente a cirurgia de bypass gástrico é o procedimento mais comumente realizado para o tratamento da obesidade mórbida nos Estados Unidos, seja por via aberta ou por laparoscopia ${ }^{1}$. No entanto, as alterações anatômicas criadas por esta cirurgia representam um grande desafio aos endoscopistas quando o acesso à árvore biliar ou ao estômago excluso é necessário ${ }^{2}$. Há poucos relatos na literatura internacional que descrevem o manejo endoscópico de doenças do trato biliar com sucesso nesses pacientes ${ }^{2,4}$. Este artigo relata o acesso laparoscópico transgástrico para o tratamento endoscópico de coledocolitíase em paciente previamente submetido a bypass gástrico em Y-de-Roux para obesidade mórbida.

\section{RELATO DO CASO}

Paciente de 52 anos, sexo masculino, submetido a bypass gástrico em Y-de-Roux por via aberta em abril de 2001 para o tratamento de obesidade mórbida $($ Peso $=140 \mathrm{~kg}$; altura $=1,80 \mathrm{~m}$; índice de massa corporal, IMC $=43,2 \mathrm{~kg} / \mathrm{m}^{2}$ ), procurou nosso serviço em dezembro de 2005 com queixa de dor abdominal em hipocôndrio direito, febre, náuseas e vômitos. $\mathrm{Na}$ ocasião seu peso era de $95 \mathrm{~kg}$, com IMC de $29,3 \mathrm{~kg} / \mathrm{m}^{2}$.

Ao exame físico apresentava-se em regular estado geral e ictérico, com dor à palpação na região subcostal direita, com sinal de Murphy positivo. Os exames laboratoriais demonstraram quadro clínico compatível com icterícia obstrutiva, com aumento dos valores de fosfatase alcalina, gama-glutamil transferase e bilirrubinas (predomínio de bilirrubina direta).

A operação proposta foi uma colecistectomia laparoscópica com colangiografia intra-operatória. A colecistectomia foi realizada utilizando duas punções de $10 \mathrm{~mm}$, uma em região supra-umbilical para a ótica de $30^{\circ}$ e outra em hipocôndrio esquerdo, e duas punções de $5 \mathrm{~mm}$, em região subcostal direita na linha hemiclavicular e em flanco direito. Após identificação do ducto cístico, um clipe foi posicionado proximalmente e o ducto foi aberto parcialmente para a introdução de um cateter para a realização da colangiografia intraoperatória, que demonstrou uma obstrução sugestiva de litíase no colédoco terminal, sem passagem do contraste para o duodeno (Figura 1A).

Como o paciente havia sido submetido a bypass gástrico, a colangiografia endoscópica retrógrada transoral não pôde ser realizada. Optou-se por realização de colangiopancreatografia endoscópica retrógrada e papilotomia por acesso transgástrico.

O estômago excluso foi mobilizado e levado à parede abdominal. A exteriorização gástrica foi realizada através da ampliação do orifício da punção subcostal esquerda de $10 \mathrm{~mm}$. A abertura da parede gástrica a céu aberto (Figura 2A) permitiu a passagem do coledocoscópio e o acesso à papila duodenal para a realização da papilotomia endoscópica. Um cálculo da via biliar pôde ser retirado com sucesso e a colangiografia pós-papilotomia demonstrou a passagem do contraste para o duodeno (Figura 1B). A parede gástrica foi

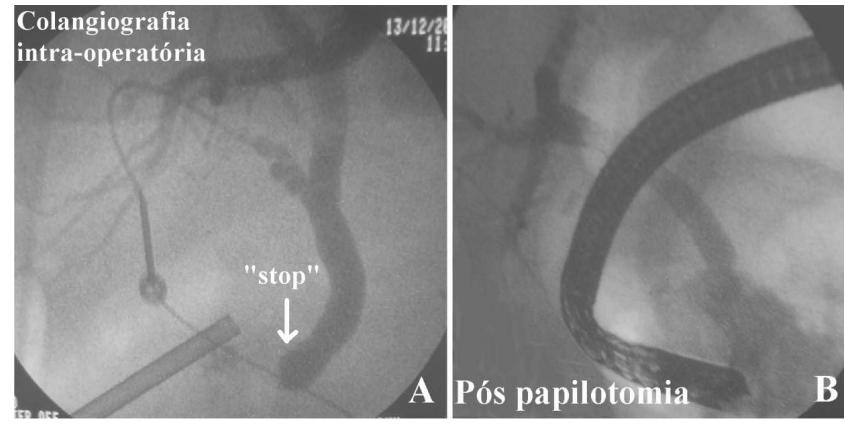

Figura 1 - (A) Colangiografia intra-operatória transcística demonstrando parada do contraste no colédoco terminal, sem progressão para o duodeno. (B) Colangiografia endoscópica retrógrada após confecção da papilotomia e retirada do cálculo, denotando a passagem do contraste para o duodeno. 
fechada em dois planos e o estômago foi re-introduzido na cavidade abdominal (Figura 2B).

O paciente apresentou excelente evolução pós-operatória, iniciando dieta via oral no $2^{\circ}$ pós operatório e recebendo alta hospitalar no $3^{\circ}$ dia após o procedimento.

\section{DISCUSSÃO}

A realização de colangiopancreatografia endoscópica retrógrada terapêutica em pacientes que foram submetidos à cirurgia com derivação em Y-de-Roux é trabalhosa, principalmente quando uma longa alça exclusa de intestino delgada foi criada ${ }^{5}$.

O primeiro caso de confecção cirúrgica de gastrostomia com o propósito único de acessar a papila para o tratamento endoscópico de doença do trato bilio-pancreático após bypass gástrico em obeso mórbido foi descrito por Baron et $a l^{3}$, em 1998. Após este relato inicial, essa via de acesso foi utilizada por outros autores para o tratamento de estenose benigna da via biliar ${ }^{4}$ e de coledocolitíase ${ }^{2}$.

A abordagem transgástrica por via laparoscópica oferece distintas vantagens incluindo o acesso rápido ao duodeno, visualização da ampola em sua anatomia usual, uso terapêutico do duodenoscópio e possibilidade de manutenção de um acesso duodenal. Ainda que a intervenção cirúrgi-

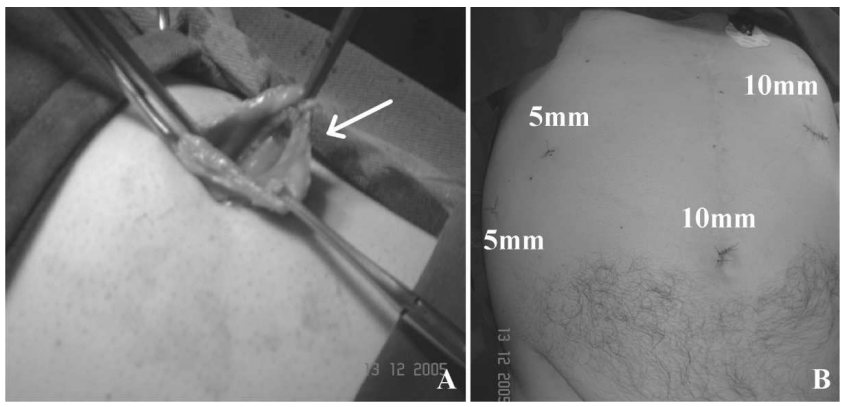

Figura 2 - (A) Exteriorização do estômago através da ampliação do orifício do trocater de $10 \mathrm{~mm}$ em hipocôndrio esquerdo para a passagem do coledocoscópio. (B) Aspecto final da cirurgia.

ca seja necessária, a gastrostomia laparoscópica está associada a uma morbidade limitada ${ }^{2}$.

Acreditamos que este seja o primeiro caso relatado no Brasil de colangiopancreatografia endoscópica retrógrada por acesso transgástrico laparoscópico para o tratamento de doenças do trato biliar após bypass gástrico em Yde-Roux em obesos mórbidos. Pudemos reproduzir com sucesso a técnica previamente descrita, confirmando a segurança desta via de acesso nesses pacientes com anatomia cirúrgica alterada.

\begin{abstract}
Access to the gastric remnant and duodenum is lost after Roux-en-Y gastric bypasses for morbid obesity. Laparoscopic transgastric endoscopic retrograde cholangiopancreatography has recently been described to manage biliary problems in such cases. We describe the first brazilian case of management of choledocholithiasis after a Roux-en-Y gastric bypass using this approach (Rev. Col. Bras. Cir. 2008; 35(6): 445-446).
\end{abstract}

Key words: Obesity morbid; Bariatric surgery/complications; Choledocholithiasis; Laparoscopic.

\title{
REFERÊNCIAS
}

1. Simpfendorfer CH, Szomstein S, Rosenthal R. Laparoscopic gastric bypass for refractory morbid obesity. Surg Clin North Am. 2005; 85(1):119-27.

2. Pimentel RR, Mehran A, Szomstein S, et al. Laparoscopy-assisted transgastrostomy ERCP after bariatric surgery: case report of a novel approach. Gastrointest Endosc. 2004; 59(2):325-8.

3. Baron TH, Vickers SM. Surgical gastrostomy placement as access for diagnostic and therapeutic ERCP. Gastrointest Endosc. 1998; 48(6):640-1.

4. Peters M, Papasavas PK, Caushaj PF, et al. Laparoscopic transgastric endoscopic retrograde cholangiopancreatography for benign common bile duct stricture after Roux-en-Y gastric bypass. Surg Endosc. 2002;16(7):1106.

5. Higa KD, Boone KB, Ho T. Complications of the laparoscopic Roux-en-Y gastric bypass: 1,040 patients - what have we learned? Obes Surg. 2000; 10(6):509-13.
Como citar este artigo:

Branco Filho AJ, Noda RW, Kondo W, George MA, Rangel M. Colangiopancreatografia endoscópica retrógrada transgástrica laparoscópica após cirurgia bariátrica Rev Col Bras Cir. [periódico na Internet] 2008; 35(6). Disponível em URL: http://www.scielo.br/ rcbc

Endereço para correspondência

William Kondo

Avenida Getúlio Vargas, 3163 ap 21

Curitiba - Paraná

E-mail: williamkondo@yahoo.com 